\title{
G3139 induces cell death by caspase-dependent and -independent apoptosis on human melanoma cell lines
}

\author{
SHINNOSUKE NAKAMURA, MADOKA INUI, TAKAHIKO KAMEI, \\ MINORU NAKASE, KENYA OKUMURA and TOSHIRO TAGAWA \\ Department of Oral and Maxillofacial Surgery, Faculty of Medicine, \\ Mie University Edobashi 2-174, Tsu city, Mie 514-8507, Japan
}

Received December 1, 2005; Accepted February 6, 2006

\begin{abstract}
G3139 is an 18-mer phosphorothioate oligodeoxynucleotide (ODN) which has been targeted on the initiation codon region of the bcl-2 gene. Currently, clinical trials on G3139 for diverse tumors are underway in phase II and phase III. However, basic investigations of bcl-2 antisense ODN (G3139) and reverse ODN (G3622) have not been fully examined. In this report, we investigate cell death caused by G3139 and G3622 and the impact of antisense ODN in melanoma cell lines. We confirmed that G3139 reduced the level of bcl-2 protein and both G3139 and G3622 inhibited cell proliferation and induced apoptosis. G3139 was noted to produce a more intense effect than G3622. Although the general caspase inhibitor, Z-VAD-fmk, prevented apoptosis incompletely, the inhibition ratio of both ODNs was approximately equivalent. Our results suggested that inhibition of cell proliferation by ODNs is produced by apoptosis, but that the apoptotic pathway is not fully induced by the caspasedependent pathway. Upon examination of the intracellular apoptotic protein dynamics, AIF localized within the mitochondria was translocated to the cytosol within $24 \mathrm{~h}$, and subsequently to the nuclei after $48 \mathrm{~h}$ of treatment with G3139. Our results imply the following: the transfection of ODNs can induce apoptosis, the anti-tumor effect of G3139 is better than G3622, and the difference in the anti-tumor effect is specifically based upon the reduction of expression of the target DNA in malignant tumors. We consider that antisense ODNs may be an important tool for anti-tumor chemotherapy and the targeting of specific DNA is important in enhancing the anti-proliferative effect against tumors.
\end{abstract}

\section{Introduction}

Overexpression of bcl-2, the anti-apoptotic protein, was confirmed in various tumor cells and its expression was related

Correspondence to: Dr Shinnosuke Nakamura, Department of Oral and Maxillofacial Surgery, Faculty of Medicine, Mie University, 2-174 Edobashi, Tsu, Mie 514-8507, Japan

E-mail: shinno@clin.medic.mie-u.ac.jp

Key words: G3139, melanoma, apoptosis, AIF in tolerance to chemotherapy and radiotherapy $(1,2)$. In melanoma cells, which are naturally resistant to various antitumor agents, bcl-2 expression was confirmed (3), and bcl-2 overexpression was related to resistance to anti-cancer chemotherapy (4). Recently, anti-tumor therapy focused on bcl-2 was examined in various cells. The impact of antisense oligodeoxynucleotide (ODN) which directly inhibits bcl-2 expression is noted. G3139 is an 18-mer phosphorothioate ODN targeted on the initiation codon region of the bcl-2 gene. G3139 has been well examined in vivo. Phase II clinical trials have been conducted for prostate cancer, breast cancer, and NonHodgkin's lymphoma, while phase III clinical trials have been performed for malignant melanoma, leukemia, and myeloma (5-9). In models of human melanoma xenografts in SCID mice, G3139 down-regulated bcl-2 protein expression and induced tumor cells to apoptosis; thus, the tumor was reduced by combination therapy with G3139 and dacarbazine (10). In clinical trial, Jansen et al reported that six of fourteen patients with metastatic melanoma responded to G3139 (11). On the other hand, G3139 exhibited no additional anti-tumor effect on combination therapy with any other anti-tumor agents. Chi et al stated that similar cytotoxic effects of G3139 were observed following overexpression of bcl-2 protein in a breast cancer cell line and that, in a similar cancer line with low bcl-2 expression, the cytotoxic activity of G3139 was not synergistic with the cytotoxic agents (12). In a melanoma cell line, the effect of G3139 combined with another cytotoxic agent was similar to that of a two base-mismatch control ODN, G4126. Benimetskaya et al (13) reported that the anti-tumor effects of G3139 and G4126 combined with Docetaxel, Thapsigargin, Gemcitibine and DDP were no different to those of the controls. Moreover, these investigators explained that the effects of a combination of Docetaxel, Thapsigargin, Gemcitibine and DDP were not apparent in cells in which bcl-2 protein was down-regulated by interfering RNA, indeed the correlation between the level of bcl-2 expression and combined effects with the cytotoxic agents was absent. Eventually, this group described that both G3139 and G4126 similarly induced cells to apoptosis and this effect of ODN was dependent upon the caspase cascade (14).

Although a phase III trial of G3139 has progressed, the competitive basic investigations on G3139 and G4126 or the reverse control ODN G3622 have been delayed. If G3139 and its mismatch or reverse control exhibit similar anti-tumor effects, the design of antisense ODN of bcl-2 would not be 
necessary. There remain controversial problems with antisense ODN therapy.

In this report, we examined whether G3139 and G3622 induced cell death in melanoma cells. Even though both ODNs induced apoptosis to the same degree, the anti-proliferative effect of G3139 was stronger than G3622. We suggest that the design of antisense for target ODNs is meaningful for anticancer agents.

\section{Materials and methods}

Cells and agents. The human malignant melanoma cell line, MMN9, was established from a submandibular lymph node metastasis of the palate melanoma. The malignant melanoma G361 cell line was purchased from RIKEN Cell Bank (Ibaraki, Japan). Both cell lines were maintained at $37^{\circ} \mathrm{C}$ in a humidified $5 \% \mathrm{CO}_{2}$ atmosphere in RPMI-1640 (Sigma, St. Louis, MO) supplemented with $5 \%$ fetal bovine serum (Sigma).

Bcl-2 antisense oligodeoxynucleotide (ODN) phosphorothioate G3139 (sequence; 5'-TCTCCCAGCGTGCGCCAT$\left.3^{\prime}\right)$ and the reverse-control ODN phosphorothioate G3622 (sequence; 5'-TACCGCGTGCGACCCTCT-3') was purchased from Calbiochem (Darmstadt, Germany). TransFectin ${ }^{\mathrm{TM}}$ (Bio-Rad, Herculs, CA) was mixed with cationic liposome and 1,2-Dioleoyl-sn-Glycero-3-Phosphoethanolamine, according to the manufacturer's protocol for transfection of both ODNs. The optimal transfection conditions were determined as the maximal dose that inhibited $<10 \%$ cell proliferation for each of the cells.

Benzyloxycarbonyl-Val-Ala-Asp fluoromethylketone (Z-VAD-fmk; Calbiochem) was used as a general caspase inhibitor.

Mouse anti-human monoclonal antibodies to bcl-2 (clone, Bcl-2-100, Oncogene, Boston, MA), p53 (clone, DO-1, Oncogene), bax (clone, 2D2, Sigma), poly(ADP-ribose)polymerase 1 (PARP-1, clone, C-2-10, Sigma) and actin (clone, JLA20, Oncogene) were used. Rabbit anti-human polyclonal antibody to apoptosis-inducing factor (AIF, Sigma) was also utilized.

Cell proliferation assay with or without ODN. Cell proliferation was assessed using the MTT assay. In brief, $5 \times 10^{3} / 50 \mu 1$ cells were seeded in 96-well plates and pre-incubated for $24 \mathrm{~h}$. The cells were then treated with 75, 150, 300 and $600 \mathrm{nM}$ ODN/100 $\mu \mathrm{l}$ with $0.3 \mu \mathrm{l}$ of TransFectin for 48 or $96 \mathrm{~h}$. Subsequently, $10 \mu 1$ of $5 \mathrm{mg} / \mathrm{ml} \mathrm{3-(4,5-dimethylthiazol-2-yl)-}$ 2,5-diphenil tetrazolium bromide (MTT) was added, and $100 \mu 1$ of $10 \%$ SDS was added $3 \mathrm{~h}$ later. After incubation for $8 \mathrm{~h}$, the absorbance at $540 \mathrm{~nm}$ was measured by enzyme immunoassay (EIA) reader (Ultrospec 3300 pro; Amersham Pharmacia Biotech AB, Uppsala, Sweden).

Western blotting. Whole cell extracts were prepared after treatment of G3139 and G3622 for the indicated time periods. The cells were harvested and washed twice with PBS. After centrifugation, cells were incubated in lysis buffer containing $100 \mathrm{mM} \mathrm{NaCl}, 1 \mathrm{mM}$ phenylmethylsulfonyl fluoride (PMSF), $1 \%$ Triton $\mathrm{X}, 0.1 \%$ SDS, $0.5 \%$ sodium deoxycholate, $20 \mu \mathrm{g} /$ $\mathrm{ml}$ aprotinin and $20 \mu \mathrm{g} / \mathrm{ml}$ leupeptin at $4^{\circ} \mathrm{C}$ for $1 \mathrm{~h}$. The protein content of the supernatant was determined using the BCA-200 protein assay kit (Pierce, Rockford, IL). After the protein concentration was adjusted to $1 \mathrm{mg} / \mathrm{ml}$, the extracts were mixed by boiling in SDS sample buffer with reducing agents and $1 \mu 1$ aliquot of the sample was applied to $10-15 \%$ SDSpolyacrylamide gradient gels for SDS-PAGE. The proteins were then electrophoretically transferred to polyvinylidene fluoride (PVDF) membranes (Immobilon-P, Millipore, Bedford, MA) using the PhastSystem (Amersham Pharmacia Biotech AB). Blotted membranes were blocked with $10 \%$ non-fat milk in TBS containing $0.1 \%$ Tween-20 (TBS-T) for $2 \mathrm{~h}$ at room temperature, and were then incubated for $1 \mathrm{~h}$ in TBS-T solution containing anti-human bcl-2, bax and PARP-1 antibodies. After incubation, the membranes were extensively washed in TBS-T and incubated with secondary horseradish peroxidaseconjugated anti-mouse IgG antibodies. Band detection was performed using the enhanced chemiluminescence system (ECL; Amersham Pharmacia Biotech UK, Little Chalfont Buckinghamshire, England Lumino-imageanalyzer; Fuji, Japan). For a protein size marker, Precision Plus Protein Standards $^{\text {TM }}$ (Bio-Rad) was used.

Each PVDF membrane detected once by a specific antibody was stripped with ReBlot Plus ${ }^{\mathrm{TM}}$ solution (Chemicon, Temecula, CA) according to the manufacturer's protocol. Subsequently, the membrane was re-probed with anti-actin antibody to confirm protein loading. The density of the detected band was measured by a chemiluminescence-image analyzer (LAS-3000 mini; Fujifilm, Japan) using MultiGauge ${ }^{\text {TM }}$ software, version 2.2. The ratio of bax/bcl-2 was calculated by density.

Detection of bcl-2 reduction. Bcl-2 protein reduction with or without ODNs was detected by immunoblotting against the bcl-2 protein. Furthermore, the quantity of bcl-2 protein with or without ODNs was measured using bcl-2 enzyme-linked immunosorbent assay (ELISA) kit (Oncogene) according to the manufacturer's protocol with a bcl-2 protein standard.

Detection of apoptosis. Detection of apoptosis was performed using the following three methods: i) DNA electrophoresis: cells treated with G3139 or G3622 for 48 h were harvested by trypsinization and washed with cold PBS. After centrifugation, the pellet was resuspended in $100 \mu 1$ cell lysate buffer [10 mM Tris- $\mathrm{HCl}$ (pH 7.4); 10 mM EDTA (pH 8.0); 0.5\% Triton X] for $10 \mathrm{~min}$. Crude lysates were centrifuged at $16000 \mathrm{rpm}$ for $5 \mathrm{~min}$ at $4^{\circ} \mathrm{C}$, and Rnase $(2 \mu \mathrm{l})$ was added to the supernatant for $1 \mathrm{~h}$ at $37^{\circ} \mathrm{C}$. Poteinase $\mathrm{K}(2 \mu \mathrm{l})$ was added for $30 \mathrm{~min}$ at $50^{\circ} \mathrm{C}$. Lysates were treated with $\mathrm{NaCl}(0.5 \mathrm{M})$ and isopropanol $(50 \%)$ at $-20^{\circ} \mathrm{C}$ overnight. After centrifugation at $16000 \mathrm{rpm}$ for $20 \mathrm{~min}$, pellets were treated with $20 \mu 1$ TE buffer [10 mM Tris-HCl (pH 7.4); 10 mM EDTA (pH 8.0)] and $4 \mu \mathrm{l}$ gel loading buffer $[0.25 \%$ bromphenol blue; $40 \%$ sucrose], and then subjected to electrophoresis in $2 \%$ agarose gel. The gel was then soaked in $0.01 \% \mathrm{SYBR}^{\mathrm{TM}}$-Green I (Molecular Probes, Eugene, OR) for $10 \mathrm{~min}$ and bands were detected by a Visi-Blue ${ }^{\mathrm{TM}}$ Transilluminator (UVP laboratory products, Upland CA). For the positive control, MMN9 cells and G361 cells, which were induced to apoptosis with anti-Fas antibody CH-11 and human recombinant IFN- $\gamma$ (Imunomax ${ }^{\mathrm{TM}}$ $\gamma$, Shionogi, Osaka, Japan), were used (15). ii) Western blotting: detection of an $85-\mathrm{kDa}$ cleavage band of PARP-1 was 
investigated for apoptosis. iii) ELISA: DNA fragmentation was detected using a cell death detection ELISA PLUS kit (Roche, Basel, Switzerland) according to the manufacturer's protocol. Z-VAD-fmk $(50 \mu \mathrm{M})$ was added $1 \mathrm{~h}$ before treatment with G3139 and G3622.

Subcellular fractionation. For determination of the intracellular dynamics of the target protein, cells treated with or without ODNs were fractionated subcellularly. Fractionation was performed using a mitochondrial fractionation kit (ACTIVE MOTIF, Carlsbad, CA) according to the manufacturer's protocol with a slight alteration. Initially, the cell lysate was divided into the nuclear and cytosolic fractions, and the secondary cytosolic fraction was divided into the mitochondrial and cytosolic fractions according to the manufacturer's protocol. The nuclear fraction was homogenized by drawing the sample through a thin needle 10-15 times and centrifugation at $800 \times \mathrm{g}$ for $20 \mathrm{~min}$ at $4^{\circ} \mathrm{C}$. The pellet was resolved in $20 \mu \mathrm{l}$ nuclear buffer [ $500 \mathrm{mM} \mathrm{KCl} ; 25 \mathrm{mM}$ HEPES (pH 7.8); 1 mM PMSF; $100 \mu \mathrm{M}$ Dithiothreitol; $10 \%$ glycerol] and incubated for $1 \mathrm{~h}$ at $4^{\circ} \mathrm{C}$. The supernatant was the nuclear fraction after centrifugation (16). Each fraction was subjected to electrophoresis for Western blotting. To confirm each fraction, detection with the p53 antibody for the nuclear fraction, bcl-2 antibody for the mitochondrial fraction, and PARP-1 antibody for the nuclear fraction were carried out.

Statistical analysis. All experiments were performed in triplicate and data were analyzed statistically using the Student's t-test. The significance was defined as $\mathrm{P}<0.01$.

\section{Results}

Down-regulation of bcl-2 and bax. We investigated the relation of the reduction of bcl-2 protein and the anti-proliferative effects on each of the cells, with or without G3139 and G3622. Reduction of bcl-2 expression was confirmed by G3139 but not by the reverse control, G3622. Bax, the bcl-2 antagonistic pro-apoptotic protein, was down-regulated following ODN treatment in both cell lines. Because the expression of both bcl-2 and bax was down-regulated (Fig. 1a), we calculated the bax/bcl-2 ratio based on the density of detected bands. The ratio of $>1$ was considered pro-apoptotic, while, $<1$ was anti-apoptotic. The ratio treated with G3139 was enhanced at 1.37 on MMN9 cells and 1.79 on G361 cells. However, the ratio treated with G3622 was reduced to 0.44 on MMN9 cells and 0.41 on G361 cells (Fig. 1b). Upon quantitative analysis of bcl- 2 protein by ELISA, the quantity of bcl- 2 was dropped to $37 \%$ with G3139 and to 9\% with G3622 in MMN9 cells after 48 h. In G361 cells, the quantity of bcl-2 decreased by $32 \%$ with G3139 but no change with G3622 was observed (Fig. 1c).

Dose-dependent anti-proliferative effect of G3139 and G3622. G3139 and G3622 dose-dependent inhibition of cell growth was confirmed in MMN9 cells. However, the effect of G3139 was stronger than that of G3622 (Fig. 2a). On the other hand, in G361 cells, both ODNs slightly inhibited cell proliferation, the effect of G3139 was significantly stronger than that of G3622 (Fig. 2b). The sensitivity of MMN9 to both ODNs a

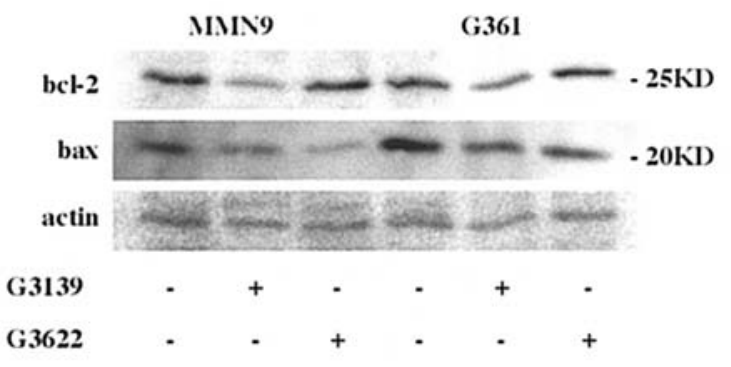

b
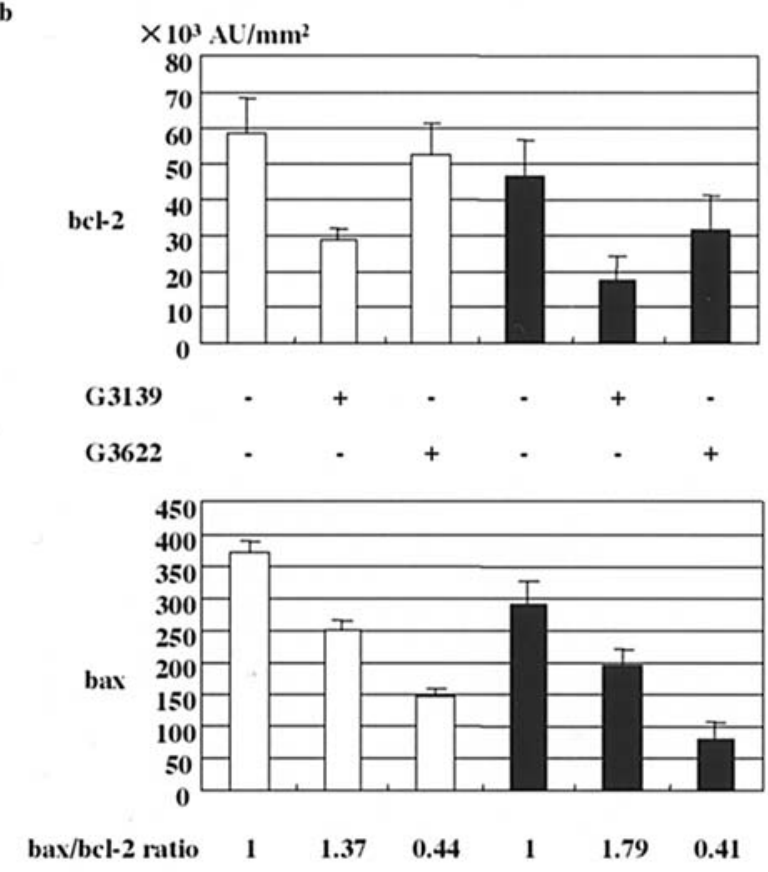

c

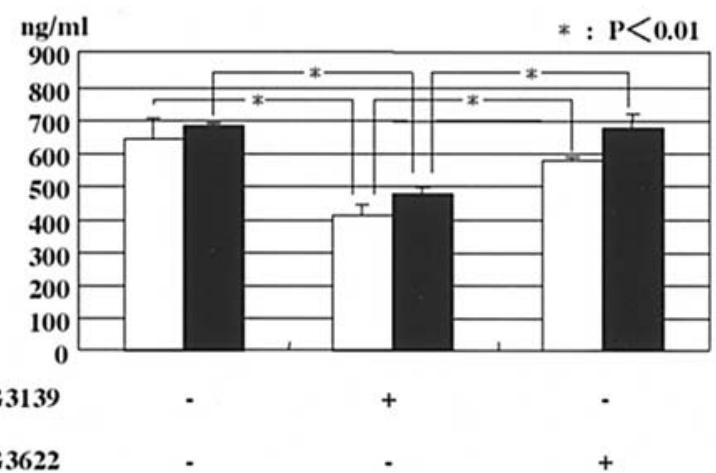

Figure 1. The expression of bcl-2 and bax with or without G3139 and G3622 using actin as a standard. (a) The expression of bcl-2 and bax is detected after treatment of $300 \mathrm{nM}$ ODNs for $48 \mathrm{~h}$. Left 3 lanes indicate MMN9 cells and right 3 lanes indicate G361 cells. (b) bax/bcl-2 ratio. The ratio is calculated based on the formula: bax/bcl-2 ratio = density of bax/density of bcl-2. The ratio is converted when the ratio of no-treatment cells is 1 . Open column, MMN9 cells; closed column, G361 cells. (c) The quantity of bcl-2 measured by ELISA after treatment of $300 \mathrm{nM}$ ODNs for $48 \mathrm{~h}$. Open column, MMN9 cells; closed column, G361 cells. ${ }^{*} \mathrm{P}<0.01$. Experiments were performed in triplicate.

was 2-fold higher than that of G361 cells. In both cell lines, treatment duration did not change the effects.

Therefore, we used $300 \mathrm{nM}$ of ODNs in the following experiments for $48 \mathrm{~h}$. The optimal dose of TransFectine for 


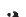

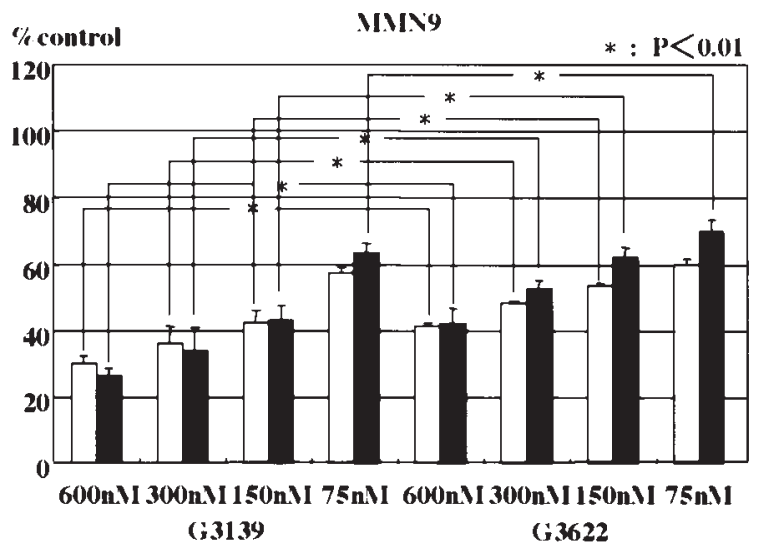

b

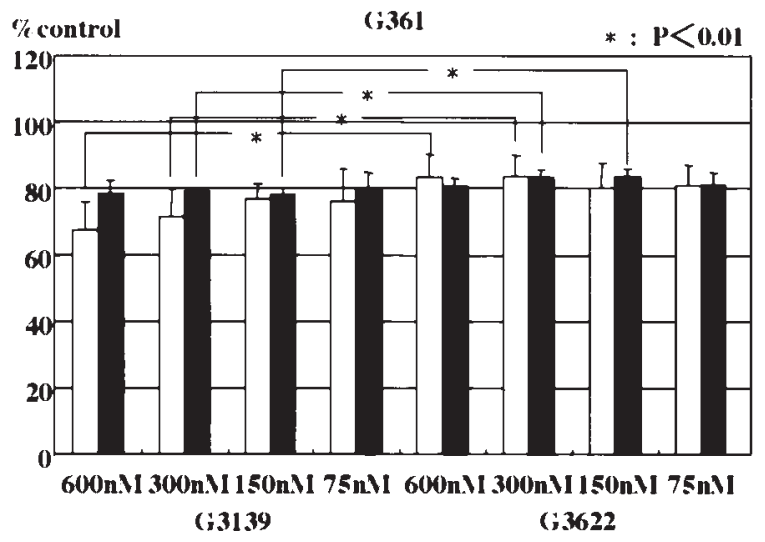

Figure 2. Dose-dependent anti-proliferative effects of G3139 and G3622. The treatment dose of ODNs are varied 600, 300, 150 and $75 \mathrm{nM}$. Open column, 48-h treatment; closed column, 96-h treatment. *Significant difference, $\mathrm{P}<0.01$. Experiments were performed in triplicate. (a) MMN9 cells: left 8 columns, G3139 treatment; right 8 columns, G3622 treatment. (b) G361 cells: left 8 columns, G3139 treatment; right 8 columns, G3622 treatment.

ODN transfection was regulated for $0.2 \%$ according to the maximal dose, with $<10 \%$ cell growth inhibition.

Detection of apoptosis by DNA ladder pattern and ELISA. We examined cell death caused by G3139 and G3622 due to apoptosis by DNA fragmentation and cleavage of PARP-1. DNA ladder patterns were apparent in both cell lines with ODNs, similar to the positive controls (Fig. 3a). Cleavage of PARP-1 was also found in both cell lines in response to both ODN and the cleavage band was greater in G3139 than G3622 (Fig. 3b). Although ELISA fragmentation assay indicated similar results of apoptosis, G3139 exhibited more induced apoptosis than G3622. Furthermore, MMN9 cells showed 2to 3-fold more apoptotic absorbance than G361 cells (Fig. 3c).

Inhibition of apoptosis with Z-VAD-fmk. Lai et al previously reported that G3139 and G4126 produced apoptosis in cells similarly and the effect was completely dependent on the caspase cascade (14). Therefore, we examined whether the apoptosis induced by both G3139 and G3622 was also dependent on the caspase cascade. Cells were pre-treated with $50 \mu \mathrm{M}$ pan-caspase inhibitor, Z-VAD-fmk, for $1 \mathrm{~h}$ and then G3139 and G3622 were transfected to the cells. In the DNA fragmentation assay, apoptosis was reduced incompletely by
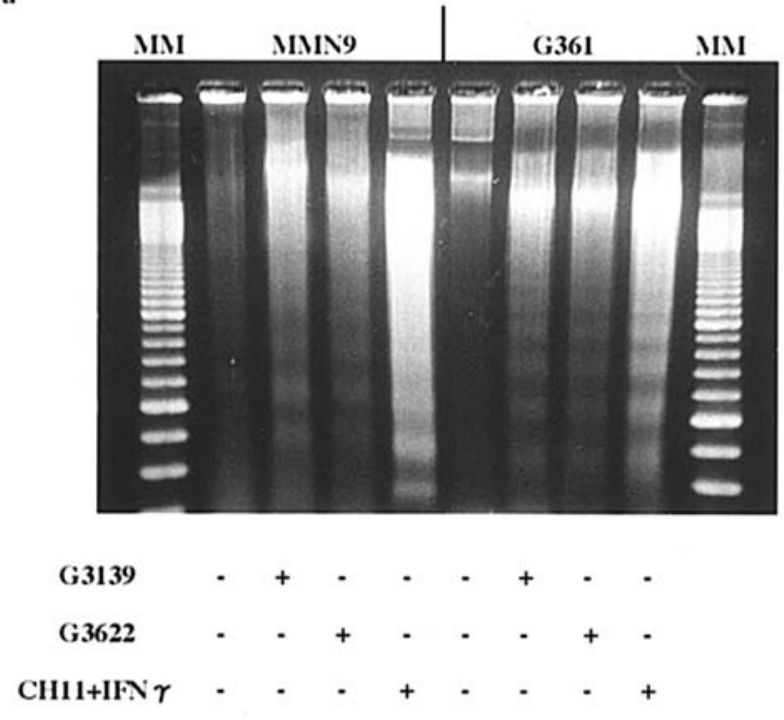

b

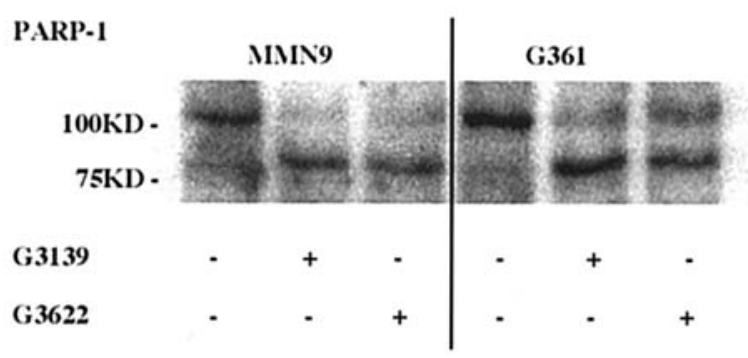

c

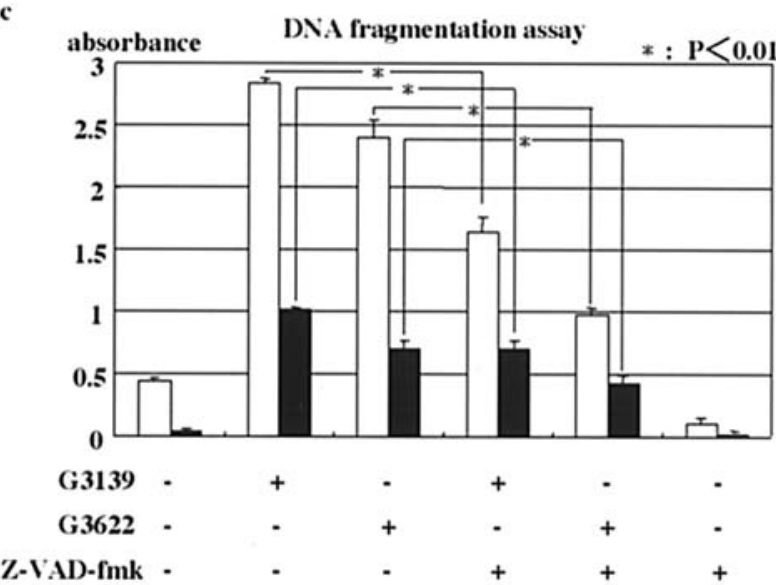

Figure 3. ODN-induced apoptosis is detected after 48-h treatment of ODNs. (a) DNA ladder pattern is detected after 48-h treatment of both $300 \mathrm{nM}$ G3139 and G3622. Combination treatment with $\mathrm{CH}-11$ and IFN- $\gamma$ is affected as positive control (17). MM, DNA size marker. (b) The cleaved PARP-1 bands indicated as $85 \mathrm{kDa}$ appeared after 48 -h treatment of $300 \mathrm{nM}$ ODNs. (c) DNA fragmentation assay. The degree of apoptosis is represented as the absorbance compared with no-treatment cells (left 2 columns). Open column, MMN9 cells; closed column, G361 cells. Z-VAD-fmk $(50 \mu \mathrm{M})$ is added $1 \mathrm{~h}$ prior to treatment with $\mathrm{G} 3139$ and G3622. ${ }^{*} \mathrm{P}<0.01$. Experiments were performed in triplicate.

Z-VAD-fmk. On G3139 treatment, the decreasing ratio of apoptosis was 30-40\% and, on G3622 treatment, the decreasing ratio was $40-60 \%$ on both cells (Fig. 3c). Attenuation of the 


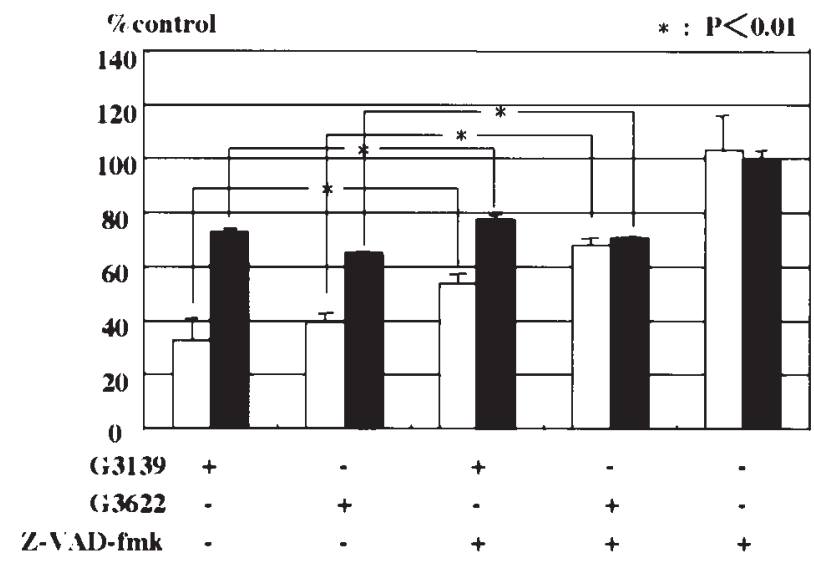

Figure 4. The anti-apoptosis effects of Z-VAD-fmk on both cell lines. ZVAD-fmk $(50 \mu \mathrm{M})$ is added $1 \mathrm{~h}$ before treatment with ODNs. Open column, MMN9 cells; closed column, G361 cells. "Significant difference, $\mathrm{P}<0.01$. Experiments were performed in triplicate.

a) MININ9 G361

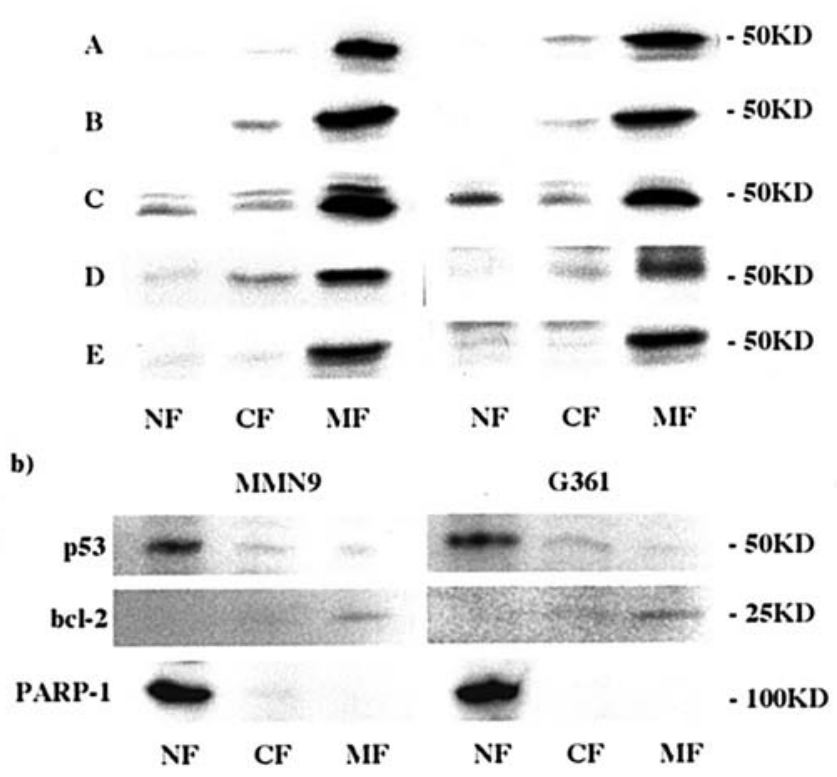

Figure 5. Intracellular dynamics of AIF. After subcellular fractionation, each fraction is subjected for immunoblotting and detection of specific antibody. (a) MMN9 and G361 cells are treated with G3139 and G3622 for $48 \mathrm{~h}$. A, no treatment; B, treated with G3139 for $24 \mathrm{~h}$; C, treated with $\mathrm{G} 3139$ for $48 \mathrm{~h}$; D, treated with G3622 for $24 \mathrm{~h}$; E, treated with G3622 for $48 \mathrm{~h}$. NF, nuclear fraction; CF, cytosolic fraction; MF, mitochondrial fraction. (b) p53, bcl-2 and PARP-1 expression on untreated MMN9 and G361 cells. NF, nuclear fraction; $\mathrm{CF}$, cytosolic fraction; MF, mitochondrial fraction.

inhibition of cell proliferation by Z-VAD-fmk was also incomplete. The effect of inhibition of cell proliferation was not restored with pre-treatment of Z-VAD-fmk (Fig. 4). The influences of Z-VAD-fmk were similar between G3139 and G3622 on both MMN9 and G361 cells.

Detection of AIF protein by subcellular fractionation. We subsequently examined the caspase-independent pathway as the caspase inhibitor did not completely inhibit apoptosis. We assessed the subcellular intracellular dynamics of AIF in the nuclear, cytosolic, and mitochondrial fractions. Fig. 5a presents the intracellular dynamics of AIF after 24- or 48-h treatment with each of the ODNs. In the MMN9 cells, AIF was recognized only in the mitochondrial fraction from the cells that were not treated (Fig. 5a, A), then it migrated to the cytosolic fraction after $24 \mathrm{~h}$ of G3139 treatment and, finally, to the nuclear fraction to induce apoptosis $48 \mathrm{~h}$ later (Fig. 5a, B and C). However, G3622 did not induce translocation of AIF. In the G361 cells, although the AIF dynamics were similar to MMN9 cells, the densities of the AIF band in the cytosolic and nuclear fractions were weak following G3139 treatment of MMN9 cells (Fig. 5a, A-E). On the control, notreatment cells, p53 and PARP-1 were detected only in the nuclear fraction, while bcl-2 was found only in the mitochondrial fraction.

\section{Discussion}

The effects of G3139 are varied and most reports have not demonstrated the effects of the reverse control, G3622, and the two base-mismatch control, G4126; therefore, the effect of antisense therapy using G3139 only is discussed. In this report, we examined the differences between the effects of G3139 and G3622 in two melanoma cell lines and investigated the inhibition of cell proliferation and cell death or apoptosis.

The inhibition of cell proliferation in MMN9 cells was greater than in G361 cells and the sensitivity to the ODNs was different between these melanoma cell lines. Although bcl-2 reduction was only confirmed on G3139 treatment, cell viability with G3622 was lowered significantly, which is likewise similar to G3139. This finding is similar to the report of Lai et al (14) that cell death was induced by ODN transfection and was not related to the degree of bcl-2 expression. Furthermore, they observed no significant differences in the inhibition of cell growth between G3139 and G4126. However, since there were no significant differences among the experimental ODNs, we consider that these differences depend on bcl-2-related cell death.

We investigated the expression of bax protein following G3139 transfection. Bax protein forms a heterodimer with bcl-2 protein; therefore, the bax/bcl-2 ratio determines the susceptibility of human melanoma cells to apoptosis (17). In this examination, bax expression was lowered concomitant with bcl-2 reduction following G3139 treatment and the bax/bcl-2 ratio was increased to 1.4-1.8 times in G3139 but decreased to $40 \%$ in G3622, compared with untreated cells.

Apoptosis is characterized by chromatin condensation and DNA fragmentation. PARP-1 is a nuclear enzyme involved in the repair of DNA damage and is cleaved by caspases during the process of apoptosis (18). We confirmed that G3139 and G3622 induced apoptosis by both DNA fragmentation assay and detection of cleavage of PARP-1 protein. Using DNA fragmentation assay, G3139 stimulated apoptosis greater than G3622 and this difference was considered to be caused by the effect of bcl-2 antisense and reverse ODNs. Furthermore, the induction of apoptosis and cleavage of PARP-1 in the MMN9 cells was greater than that in G361 cells, similar to the result of prevention of cell proliferation.

In the examination of whether apoptosis was dependent upon the caspase cascade, apoptosis was attenuated with 
Z-VAD-fmk and cell death was not fully restored following Z-VAD-fmk pretreatment. However, the inhibition rate depending upon Z-VAD-fmk was similar between G3139 and G3622 treatments. Recently, two distinct pathways leading to nuclear apoptosis have been reported. One of the pathways leads to oligonucleosomal DNA fragmentation in a caspasedependent manner, whereas the other pathway acts directly on the mitochondria and induces apoptosis in a caspaseindependent manner. Therefore, we studied the incidence of other mechanisms of cell death, excepting caspase-dependent apoptosis. For a factor that directly induced apoptosis, AIF has been reported. AIF, which is a mitochondrial protein, moves to the cytosol and nuclei following apoptosis-inducing stimuli and can lead to cell death as a result of cell death-induced chromatin aggregation and large-size DNA fragmentation $(19,20)$.

We next investigated the AIF location within subcellular fractions, with or without treatment of G3139 and G3622, for 24 and 48 h. Upon treatment of G3139, AIF translocated from the mitochondria to the cytosol $24 \mathrm{~h}$ later and moved to the nuclei $48 \mathrm{~h}$ later in both cell lines. However, G3622 exhibited no movement of the AIF from the mitochondria in either cell lines.

There has been one report that bcl-2 down-regulation results in autophagy in a caspase-independent manner in HL60 cells. Saeki et al reported that bcl-2 antisense ODNs induce translocation of the AIF to the nuclei in an autophagic fashion, not apoptosis. Therefore, bcl-2 antisense ODNs do not induce a DNA ladder pattern and cannot be rescued by caspase inhibitors (21). Overexpression of bcl-2 inhibits the early caspase-dependent onset of apoptosis but does not prevent the late stage of caspase-independent apoptosis by AIF (22). In this report, a ladder pattern is observed by G3139 and G3622 and AIF is translocated by G3139. Whether bcl-2 attenuation of bcl-2 antisense ODN stimulates the effect(s) of AIF or antisense ODNs directly enhance AIF has not been confirmed. Thus, further investigation is necessary to elucidate the mechanism(s).

In conclusion, we demonstrated that ODN which is not restricted antisense ODN can induce apoptosis in melanoma cells. Cell death by antisense ODN is dependent not only on caspase but also on AIF which is caspase-independent. The G3139 anti-tumor effect and induction of apoptosis was stronger than G3622 and the advantage of the anti-tumor effect is based upon targeting bcl-2. Therefore, we consider that antisense therapy with targeting of tumor-specific DNA will be significant for future anti-tumor chemotherapy using antisense ODN.

\section{Acknowledgements}

Part of this study was supported by a grant-in-aid for Science Research from the Japan Society for the Promotion of Science.

\section{References}

1. Daidone MG, Luisi A, Veneroni S, Benini E and Silvestrini R: Clinical studies of bcl-2 and treatment benefit in breast cancer patients. Endocr Relat Cancer 6: 61-68, 1999.
2. Miyake H, Hanada N, Nakamura H, et al: Overexpression of bcl-2 in bladder cancer cells inhibits apoptosis induced by cisplatin and adenoviral-mediated p53 gene transfer. Oncogene 16: 933-943, 1998.

3. Cerroni L, Soyer HP and Kerl H: Bcl-2 protein expression in cutaneous malignant melanoma and benign melanocytic nevi. Am J Dermatopathol 17: 7-11, 1995.

4. Serrone L and Hersey P: The chemoresistance of human malignant melanoma: an update. Melanoma Res 9: 51-58, 1999.

5. Waters JS, Webb A, Cunningham D, et al: Phase I clinical and pharmacokinetic study of bcl-2 antisense oligonucleotide therapy in patients with Non-Hodgkin's lymphoma. J Clin Oncol 18: 1812-1823, 2000.

6. Rudin CM, Otterson GA, Mauer AM, et al: A pilot trial G3139, a bcl-2 antisense oligonucleotide and paclitaxel in patients with chemorefractory small-cell lung cancer. Ann Oncol 13: 539-545, 2002.

7. Chi KN, Gleave ME, Klasa R, et al: A phase I dose-finding study of combined treatment with an antisense bcl-2 oligonucleotide (Genasense) and mitoxantrone in patients with metastatic hormone-refractory prostate cancer. Clin Cancer Res 7: 3920-3927, 2001.

8. Marcucci G, Byrd JC, Dai G, et al: Phase I and pharmacodynamic studies of G3139, a bcl-2 antisense oligonucleotide, in combination with chemotherapy in refractory or relapsed acute leukemia. Blood 101: 425-432, 2003.

9. Marcucci G, Stock W, Dai G, et al: Phase I study of Oblimersen sodium, an antisense to bcl-2, in untreated older patients with acute myeloid leukemia: pharmacokinetics, pharmacodynamics and clinical activity. J Clin Oncol 23: 3404-3411, 2005.

10. Jansen B, Schlagbauer-Wadl H, Brown BD, et al: Bcl-2 antisense therapy chemosensitizes human melanoma in SCID mice. Nat Med 4: 232-234, 1998.

11. Jansen B, Wacheck V, Heere-Ress E, et al: Chemosensitisation of malignant melanoma by bcl-2 antisense therapy. Lancet 356 : 1728-1733, 2000.

12. Chi KC, Wallis AE, Lee CH, et al: Effects of bcl-2 modulation with G3139 antisense oligonucleotide on human breast cancer cells are independent of inherent bcl-2 protein expression. Breast Cancer Res Treat 63: 199-212, 2000.

13. Benimetskaya L, Lai JC, Khvorova A, et al: Relative bcl-2 independence of drug-induced cytotoxicity and resistance in 518A2 melanoma cells. Clin Cancer Res 10: 8371-8379, 2004.

14. Lai JC, Benimetskaya L, Khvorova A, et al: Phosphorothioate oligodeoxynucleotides and G3139 induce apoptosis in 518A2 melanoma cells. Mol Cancer Ther 4: 305-315, 2005.

15. Kamei T, Inui M, Nakamura S, Okumura K, Goto A and Tagawa T: Interferon- $\gamma$ and anti-Fas antibody-induced apoptosis in human melanoma cell lines and its relationship to bcl-2 cleavage and bak expression. Melanoma Res 13: 153-159, 2003.

16. Corbiere C, Liagre B, Terro F and Beneytout JL: Induction of antiproliferative effect by diosgenin through activation of p53, release of apoptosis-inducing factor (AIF) and modulation of caspase-3 activity in different human cancer cells. Cell Res 14: 188-196, 2004.

17. Raisova M, Hossini AM, Eberle J, et al: The bax/bcl-2 ratio determines the susceptibility of human melanoma cells to CD95/ Fas-mediated apoptosis. J Invest Dermatol 117: 333-340, 2001.

18. Germain M, Affer EB, D'Amours D, Dixit VM, Salvesen GS and Poirier GG: Cleavage of automodified poly (ADP-ribose) polymerase during apoptosis. J Biol Chem 274: 28379-28384, 1999.

19. Susin SA, Daugas E, Ravagnan L, et al: Two distinct pathways leading to nuclear apoptosis. J Exp Med 192: 571-579, 2000.

20. Daugas E, Susin SA, ZamZami N, et al: Mitochondrio-nuclear translocation of AIF in apoptosis and necrosis. FASEB J 14: 729-739, 2000.

21. Saeki K, Yuo A, Okuma E, et al: Bcl-2 down-regulation causes autophagy in a caspase-independent manner in human leukemic HL60 cells. Cell Death Differ 7: 1263-1269, 2000.

22. Xu DZ, Susan KG and Peter H: Staurosporine induces apoptosis of melanoma by both caspase-dependent and -independent apoptotic pathways. Mol Cancer Ther 3: 187-197, 2004. 